
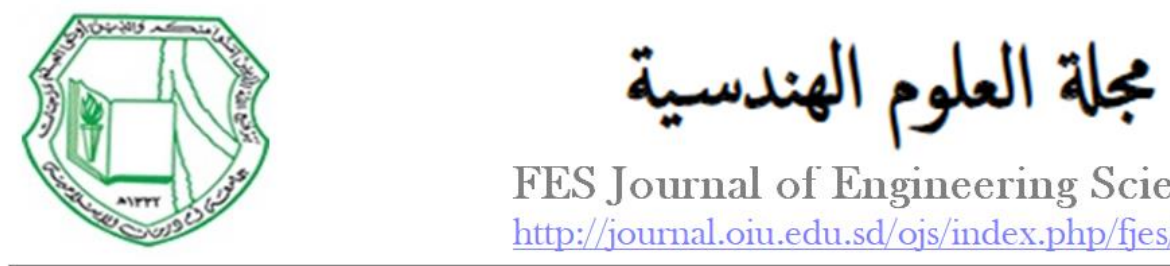

\title{
شكل المسقط الأفقي الأمثل لصالة الصلاة بالمساجد
}

\author{
أحمد الطيب حسن الفاتح قريب الله \\ قسم العمارة و التخطيط - كلية الهندسة. جامعة أم درمان الإسلامية ـ السودان (E. mail: engahmedh1@gmail.com)
}

Article history: Received 14 October 2020, Received in revised form 14 April 2021, Accepted 17 April 2021

\begin{abstract}
The plans of mosques have different shapes (rectangle, square, triangle, elliptical, octave, circular ...) depending to the designer's ideas, also the plans governed by the functions of the buildings according to the service that achieved, The main function of the mosque is to worship it in prayer and other religious lessons and adhkaar, The researcher or designer should avoid some forms of plans that are not suitable for the function of prayer rooms, and the problem of studying is the existence of some forms that are not suitable for the plan of these halls. The study aims to monitor, analyze and evaluate the forms of plans used in the mosques of the Khartoum region to determine the optimum shape in terms of the functional suitability of this important ritual, and the study was achieved according to an analytical methodology rooted in the terms of the Islamic legislation and based on the optimal standard that serves the function of the appropriate geometric shape of prayer hall plan in the mosque. According to studies of the various forms addressed by the researcher and know its pros and cons to come up with results and conclusions that achieve the objectives of the research, which is to reach the optimal and better form that serves the function of the prayer rite of stacking the ranks and performing different movements. The study concludes that the best and optimal shape of the plan reached is the rectangular shape whose longest rib perpendicular to the direction of the qibla.
\end{abstract}

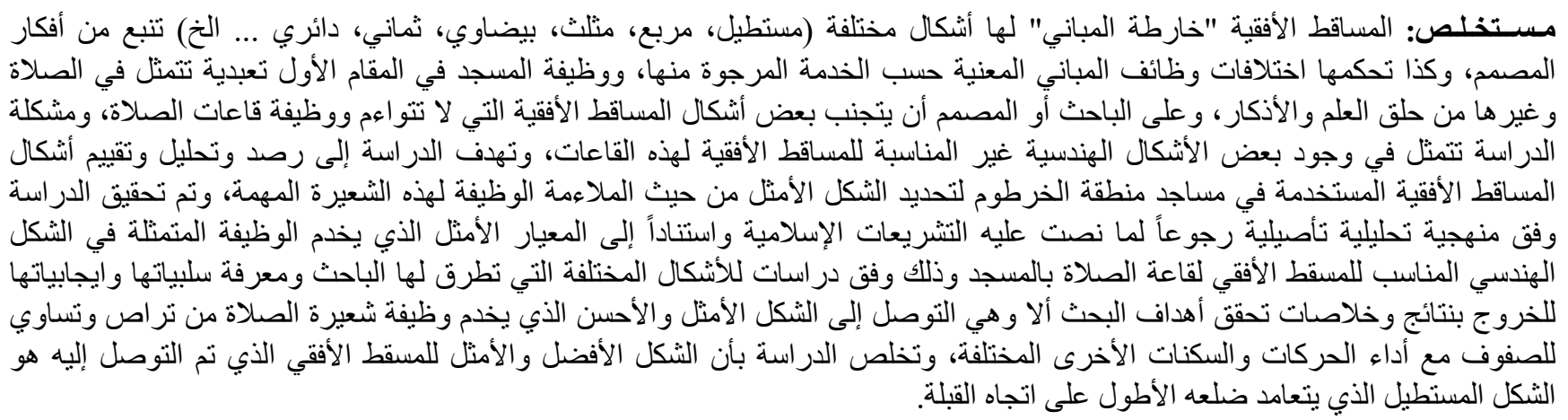


عن جابر بن سمرة رضي الله عنهما قال: خرج علينا رسول الله

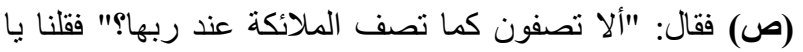
رسول الله وكيف تصف الملائكة عند ربها؟ قال: "يتمون الصف الصف

الأول ويتر اصون في الصف". [رو اه مسلم - النووي].

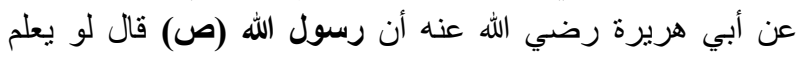

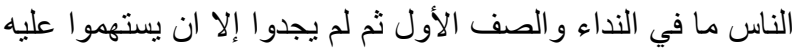
لاستهموا. [متفق عليه ـ النووي].

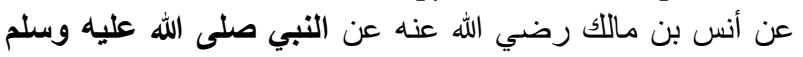

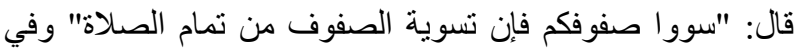
رواية البخاري "سووا صفوفكم فان تسوية الصفوف من اقامة التهان

$$
\text { الصلاة". [البخاري ومسلم]. }
$$

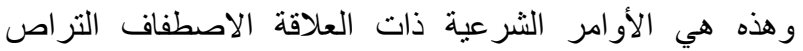

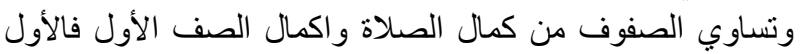

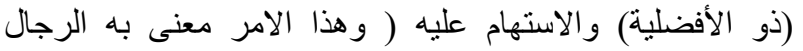
و النساء كل فى مكانه المخصص) وهي الأنهام (المعايير التي حتماً ستؤثر الأر

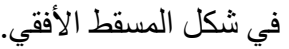

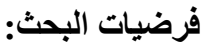

فروض الدراسة تتمثل في أن الأشكال الهندسية المختلفة للمساقط

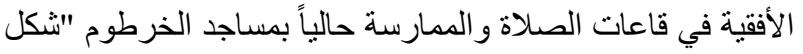

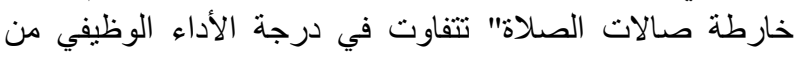

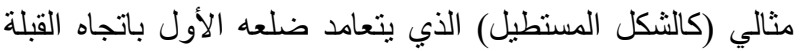

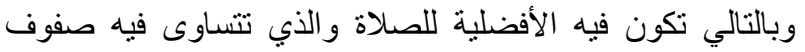

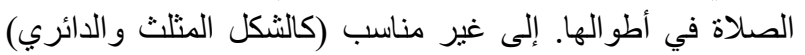

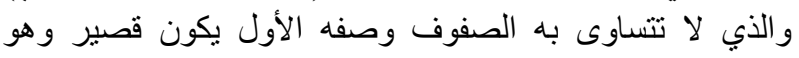
الصف ذو الأفضلية التي نصل عليها الثرع في الأحاديث

يهذف البحث: إلى دراسة الأشكال الهندسية للمساقط الأفقية

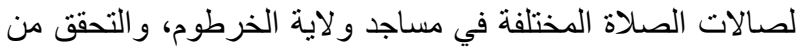

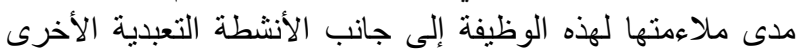

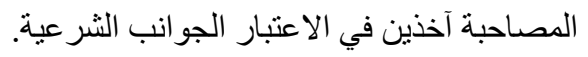

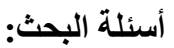

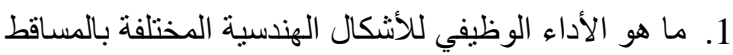

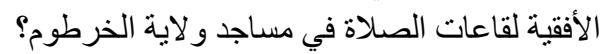

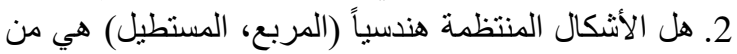

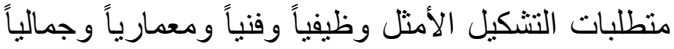

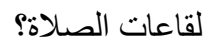

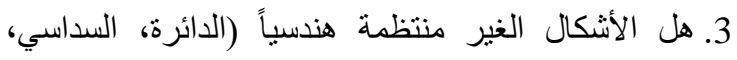

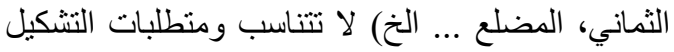

الأمثل لقاعات الصلاة (وظيفياً وفنياً ومعمارياً وجمالياً)؟

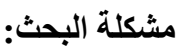

تتمتل مشكلة البحث في أن بعض الأشكال الهندسية ذات المساقط

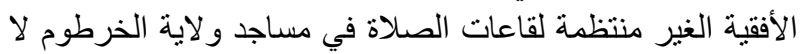

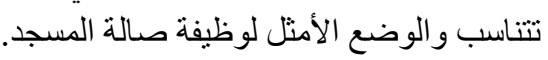

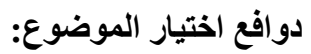

من دو افع اختبار موضوع الدارعة الداسة هو العمل على تطوير وضبط شكل المسقط الأفقي لقاعات الصلاة في مساجد السودان بصورئ الصورة
تعريف المسجد لغة واصطلاحاً:

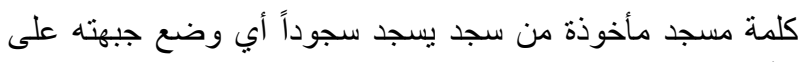
الأرض [ابن منظور ، لسان العرب].

وكل شيء ذل فقد سجد [المصباح المنير].

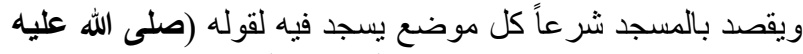
وسلم): "وجعلت في الأرض مسجداً وطهور أ"، [صحيح البخاري]

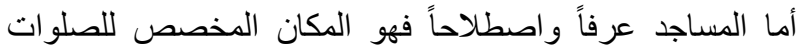

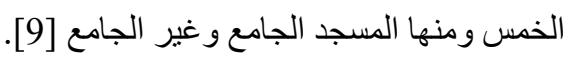

\section{تمهيد:}

إن أهم ركن من أركان الدين هو الصلاة، قال تعالى (إنَّ الصَّلأَة

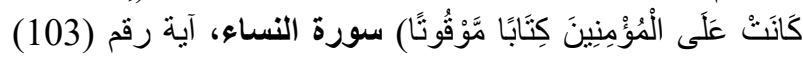

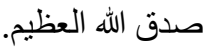

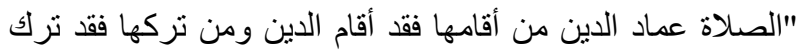

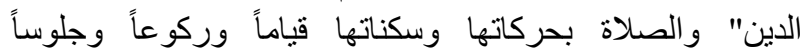

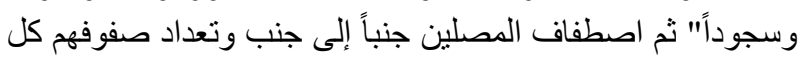

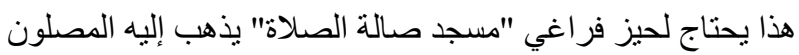

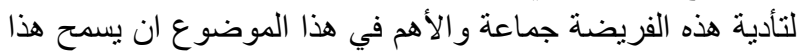

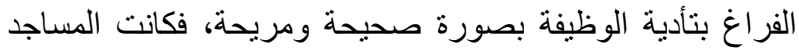

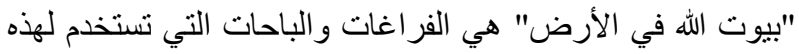

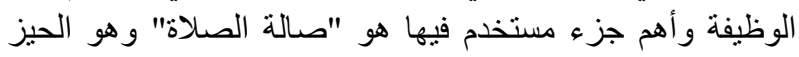

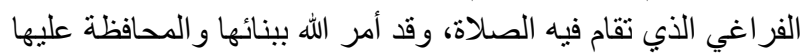
وو عد باللثواب والأجر لذللك فقيل في الحديث: "من بنى الله لله مسجداً

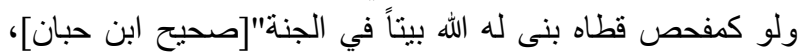
إذن فهي من المباني المهمة جداً. الحيز الداخلي هو ذللك الجزء الذي الذي اقتطعه الإنسان من الفراغ العان العام

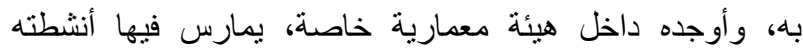

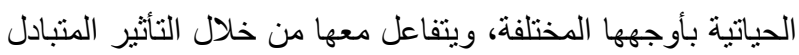

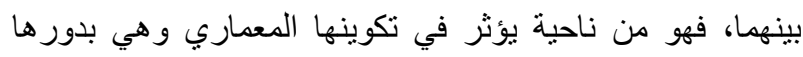

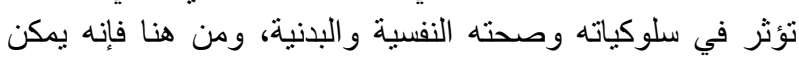

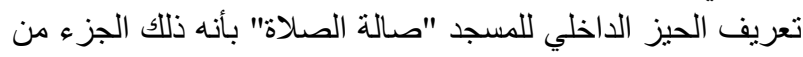

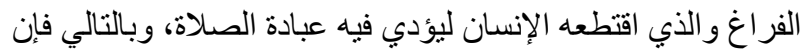

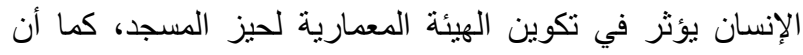

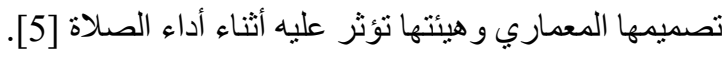

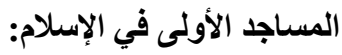

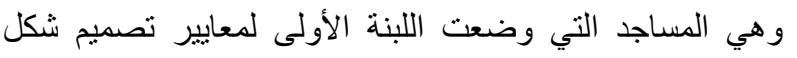

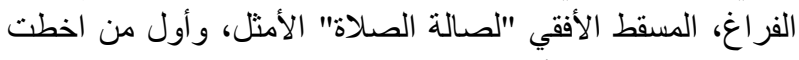

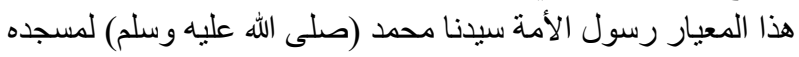
بالمدينة المنورة ووقف عليه منذ أن كان أرضاً خاوية ثم بدأ البناء

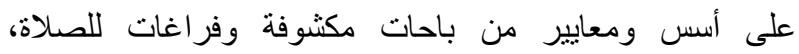

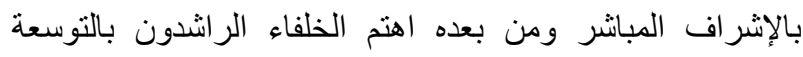

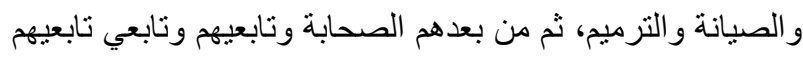

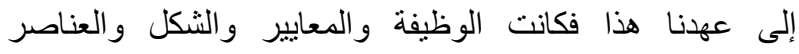

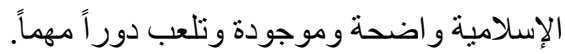

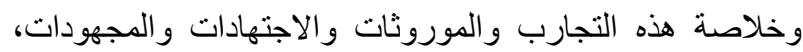
تمثلت في الأشكال و الكتل و الفراغات ذات ذات الطابع الإسلامي المتميز

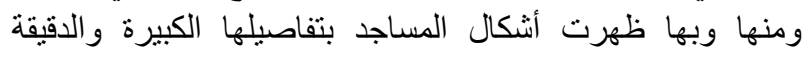

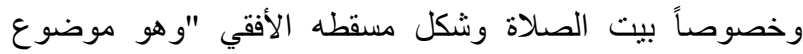

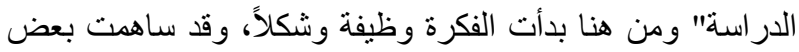
الأحاديث النبوية في التشكيل المعماري الفر اغي لبيوت النيات الصلاة. 


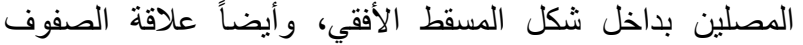

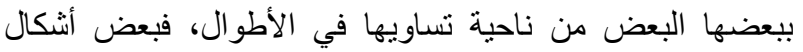

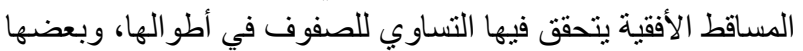

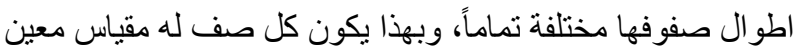

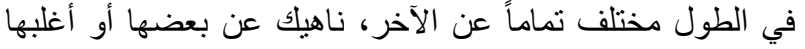

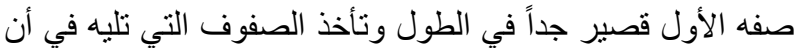

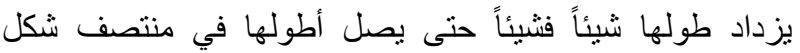

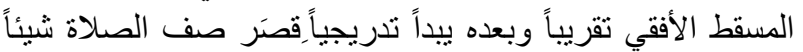

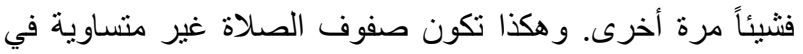

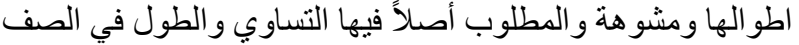
الأول، و هو الوضع الأمثل و المطلوب.

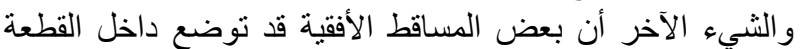
الممنوحة لإنشاء المسجد وأحد اضلاعه لا لا تكون عمودية على اتجاه

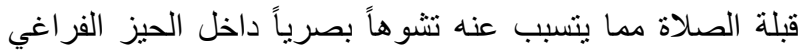

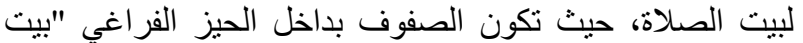
الصلاة" غير موازية لإحدى الحوائط ولكن عمودية على التهاه القبلة وبعضها لا يجد فيه المصلون رازية راحة الرؤية الداخلية وذلك الكاه

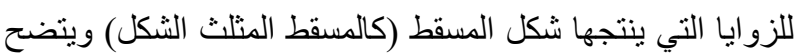
هذا جلياً في بعض الأشكال التالية (الثكل رقم (1) إلى الثنكل رقم و هي الأشكال التي تخيلها الدارس ليوضح بها الخلل و عدم التساوي

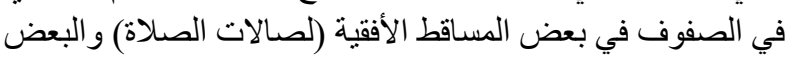
الآخر المناسب شر عاً ووظيفة.

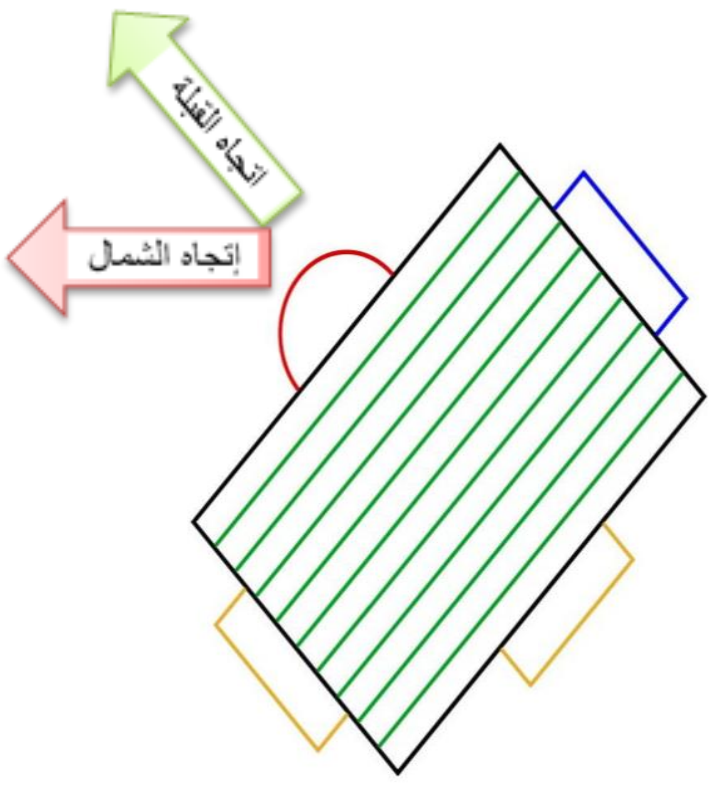

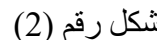

شكل المسقط الافقي لصالة الصلاة مستطيل الشكل

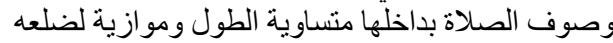
الطويل و عمودية على اتجاه القبلة المصدر : الباحث علث أن
عامة ومساجد و لاية الخرطوم بصفة خاصة، وذللك لتحقيق الأداء الأمثل وظيفياً وفنباً ومعمارياً وجمالياً.

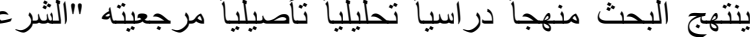

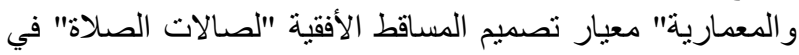
المساجد وذللك بدراسة الأثكال المختلفة لها وتحليلها ومعرفة الهية

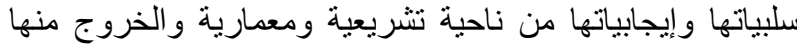

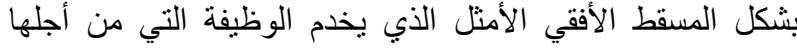

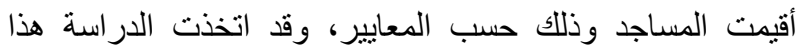

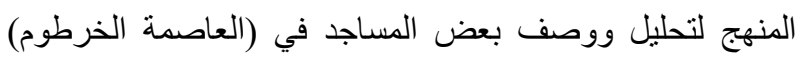

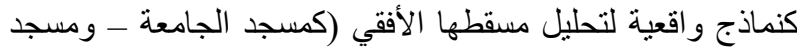
النيلين). (- ماذج)

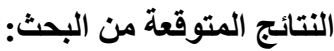

النتائج المتوقعة لهذه الدراسة تنتثثل في التوصل إلى الثنكل

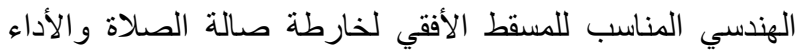

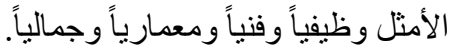

\section{أثكال المساقط الأفقية المختلفة لبيوت الصلاة}

تمهيد: يستعرض البحث هنا بعض الأشكال للمساقط الأفقية "لصالات

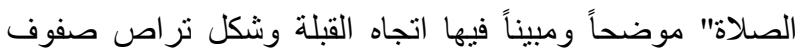
الصلاة بداخلها ويرى فيها جلياً علاقة صفوف الصلاة وتناة وتراص

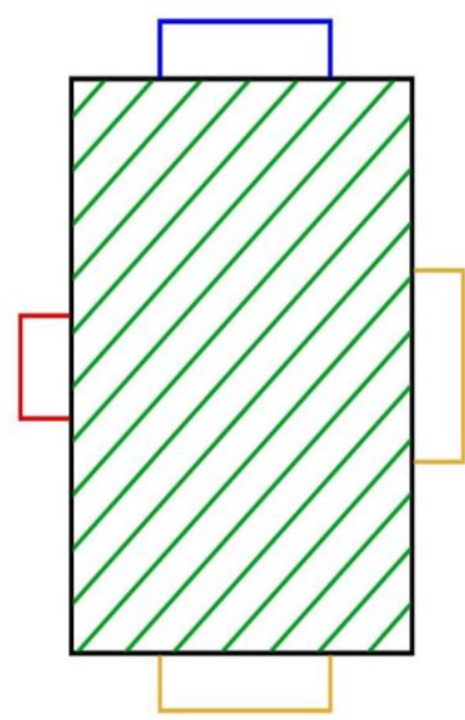

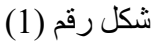

شكل المسقط الافقي لصالة الصلاة مسنطيل الثكل وصلي وصوف الصناة بداخله غير منساوية الطول و غير موازية للحو ائط ولكنها عمودية الطية على اتجاه القبلة المصدر : الباحث المثة 


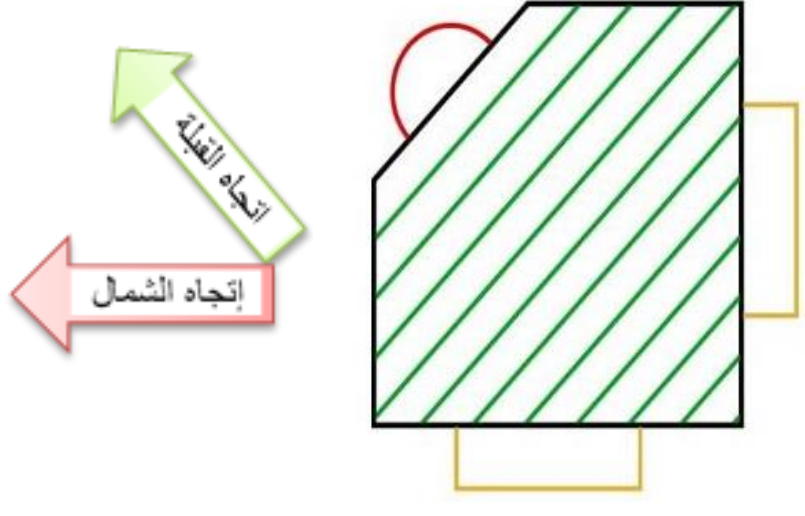

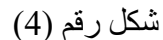

شكل المسقط الافقي لصالة الصلاة مربع الثكل وصفوف الصلاة بداخلها غير منساوية الطول وغير موازية للحو ائطو لكنها عمودية

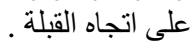

المصدر: الباحث

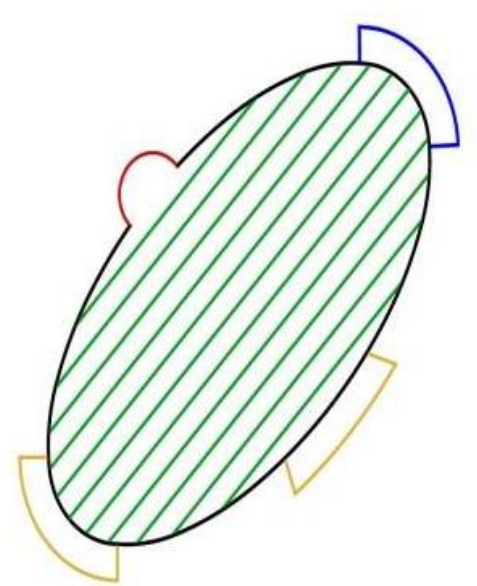

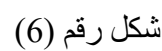

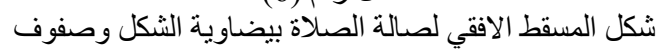
الصلاة بداخلها غير متساوية الطول و عمودية على اتجاه القبلة المصدر : الباحث

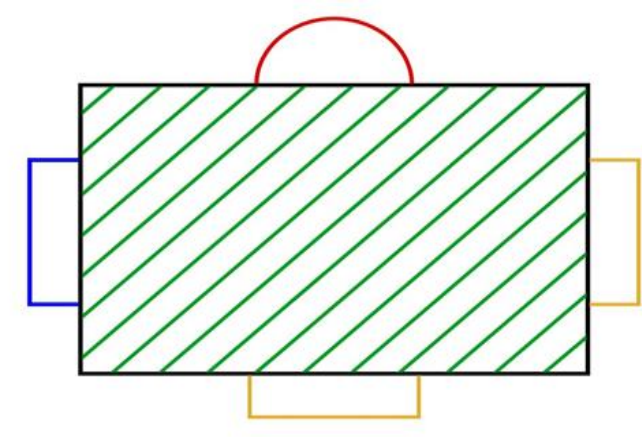

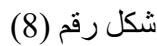

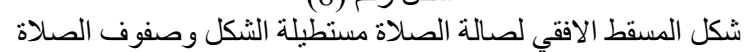

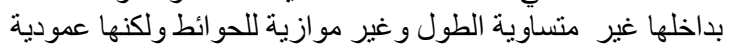
على اتجاه القبلة

المصدر: الباحث

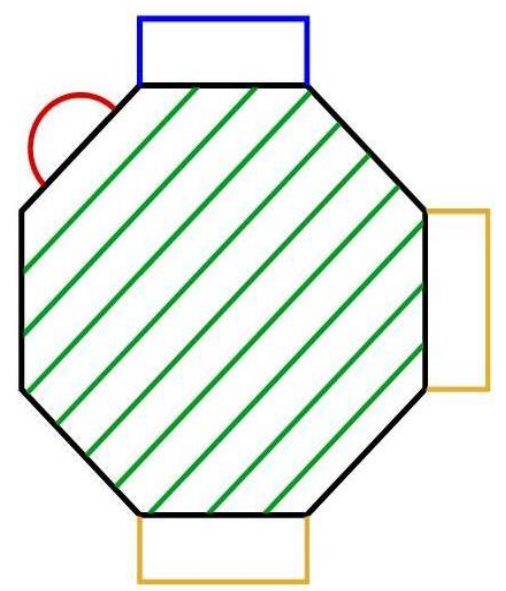

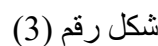

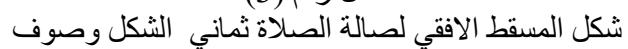

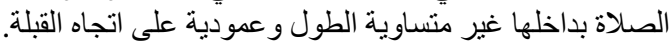
المصدر: الباحث

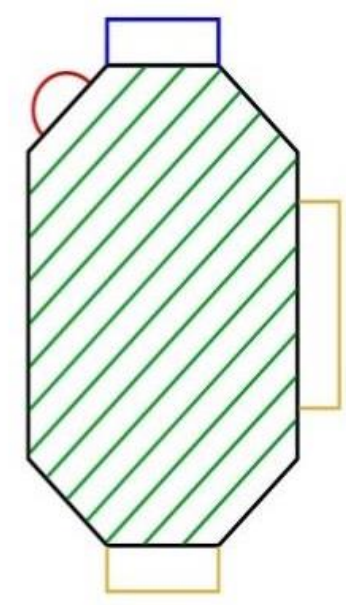

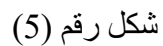

شكل المسقط الافقي لصالة الصلاة ثماني الثكل باسنطالة شرقية غربية وصفوف الصلاة بداخلها غير متساوية الطول وعمودية على بلى اتجاه القبلة

المصدر : الباحث

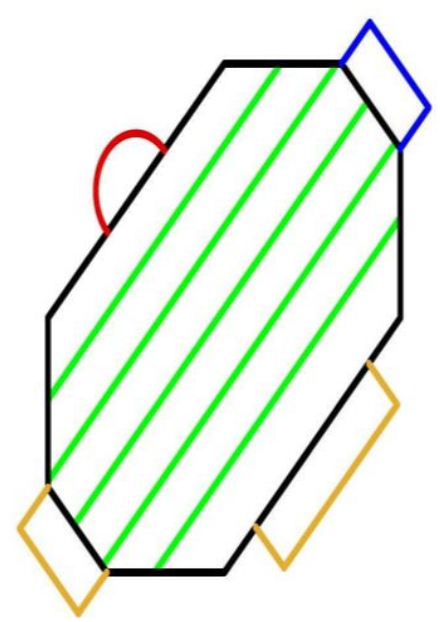

شكل رقم (7)

شكل المسقط الافقى لصالة الصنلاة ثماني الثكل باستطالة (جنوبية

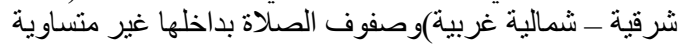
الطول و عمودية على اتجاه القبلة المصدر : الباحث عمثان 


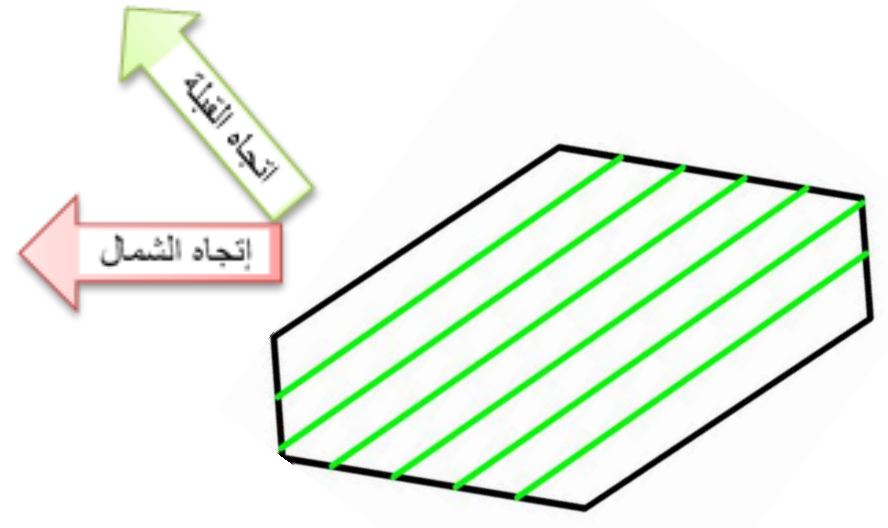

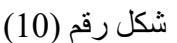

شكل المسقط الافقي لصالة الصلاة سداسية الثكل بباستطالة (جنوبية شرقية - شمالية غربية ) وصوف الصلاة بداخلها غير لئل متساوية الطول و وعمودية على اتجاه القبلة

المصدر : الباحث

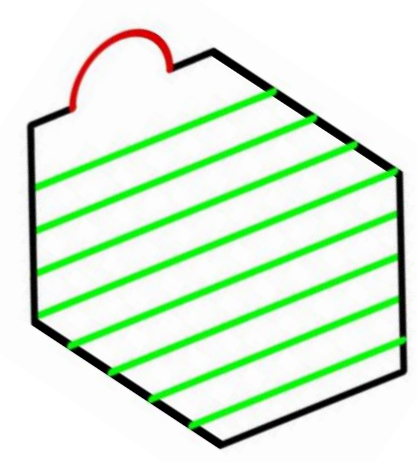

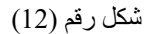

شكل المسقط الافقي لصالة الصنلاة سداسي منتظم الثكل وصفوف الصلاة لداخله غير متساوية الظول و عمودية على اتجاه القبلة

المصدر : الباحث

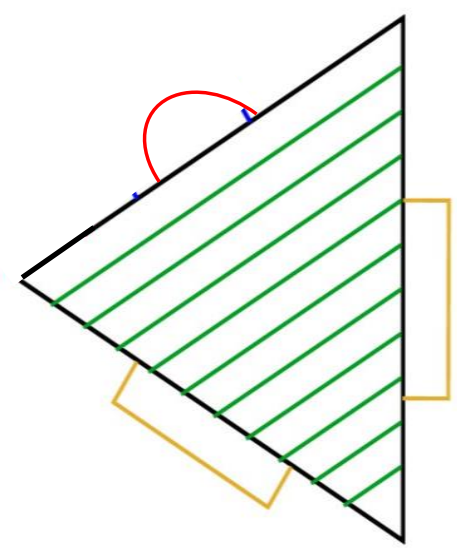

شكل رقم (14) (14)

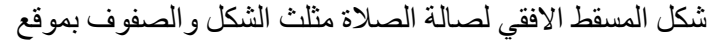

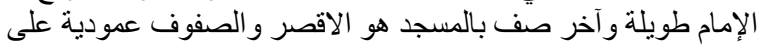

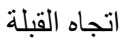

المصدر : الباحث

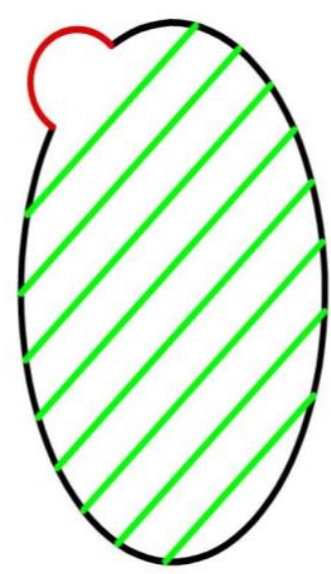

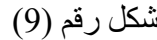

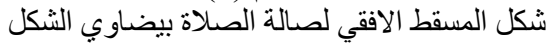

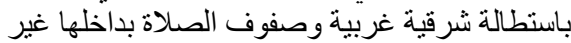
متساوية وعمودية على اتجاه القبلة القبلة المصدر : الباحث

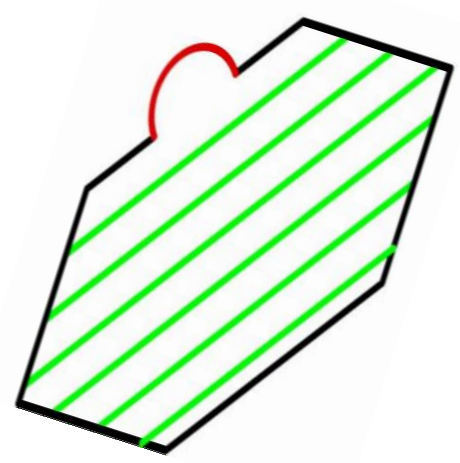

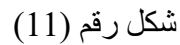

شكل المسقط الافقي لصالة الصلاة سداسي الثكل باستطالة (جنوبية

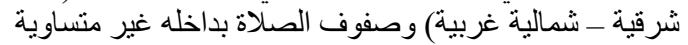
و عمودية على اتجاه القبلة

المصدر: الباحث آتياه

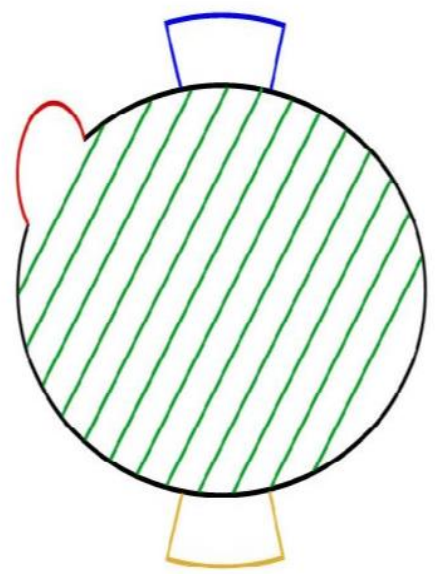

شكل رقم (13)

شكل المسقط الافقي لصالة الصلاة دائرية الثكل وصفوف

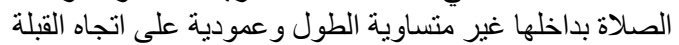
المصدر: الباحث 


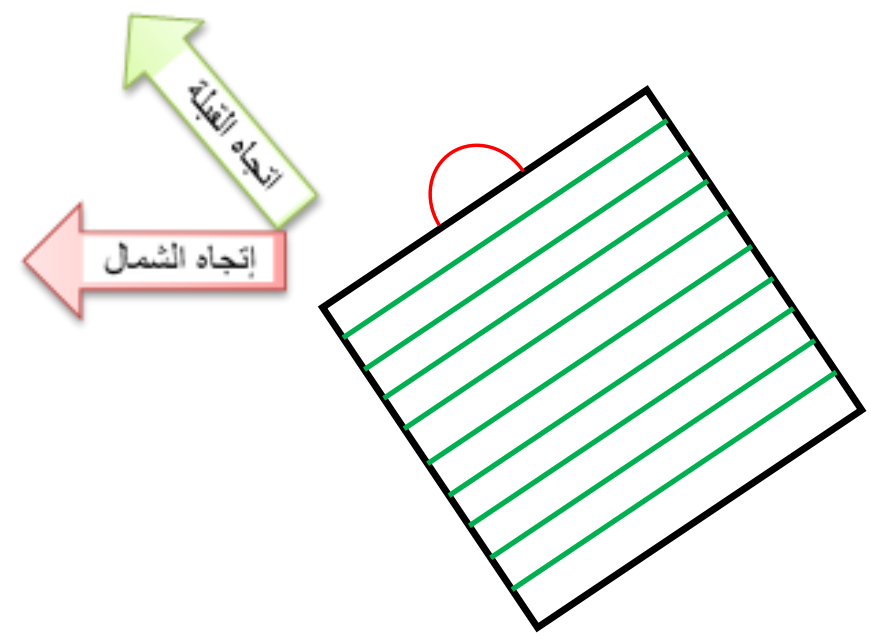

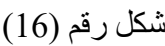

شكل المسقط الاقفي لصالة الصلاة مربع الثكل وصفوف

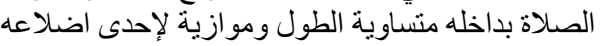

و عمودية على اتجاه القبلة لاعه

$$
\text { المصدر: الباحث }
$$

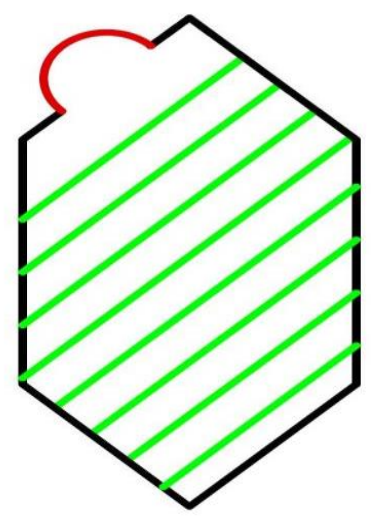

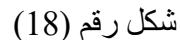

شكل المسقط الافقي لصالة الصلاة سداسية الثكل باستطالة شرقية غربية وصفوف الصلاة بداخلها غير متساوية الطول و عمودية على الثى اتجاه القبلة

المصدر: الباحث

الحرام (الكعبة المشرفة) والكعبة هنا تتوسط صحن المسجد و الصفوف تكون دو ائر حولها، وكذللك يلاحظ في الثنكل رقدم (21)

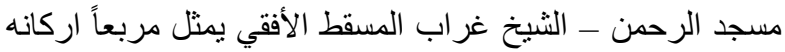

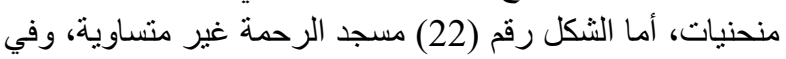

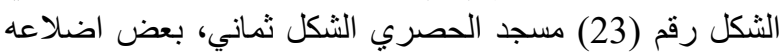

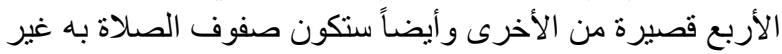

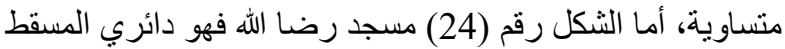
وستكون بداخله صفوف الصلاة غير متساوية، ويظهر بهذا جلئائا التباين في الأشكال وما سيترتب على كل شكل من تنظيم نساوي

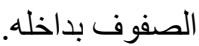

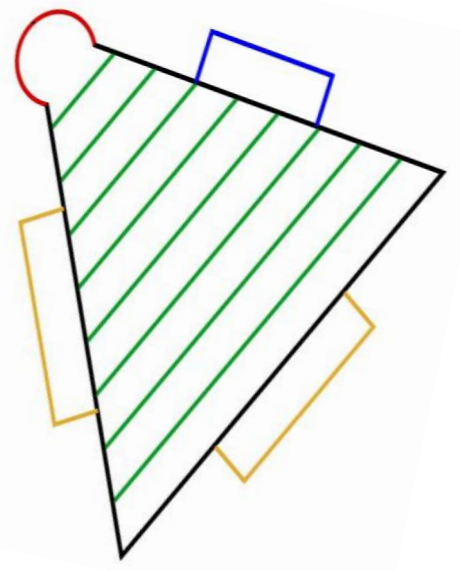

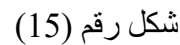

شكل المسقط الافقي لصالة الصلاة مثلث الثكل و الصفوف

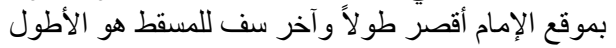
و الصفوف عمودية على اتجاه القبلة المصدر: الباحث

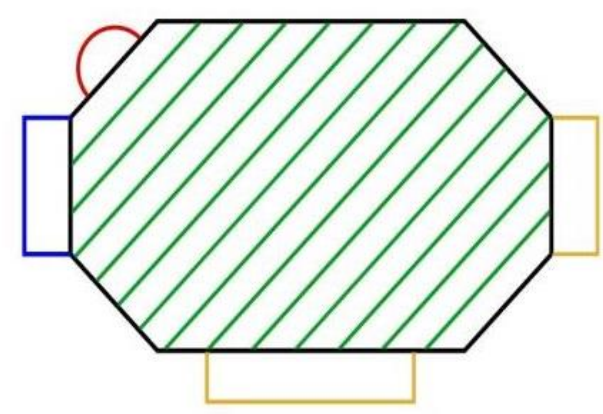

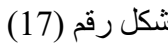

شكل المسقط الافقي لصالة الصلاة ثماني الثكل باستطالة شمالية جنوبية وصفوف الصلاة غير متساوية الطول و عمودية على الطى

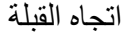
المدر: الباحث

أشكال مساقط أفقية لبعض المساجد: ويستعرض البحث هنا بعض الأشكال (من الثكل رقم (19) إلى الى

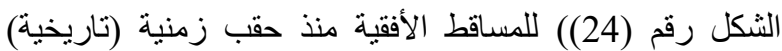
قديمة للمساجد الأولى في صدر الإسلام وبعضها حديث، ويرى الإنه واضحاً اختلاف الأشكال وما نتج عند الأنها من سلبيات وايجابيات تحدث عنها البحث. يوضح الثكل رقم (19) المسجد النبوي و التوسعة التي جرت عبر التبر

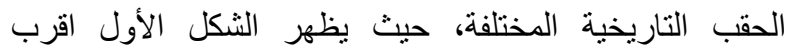

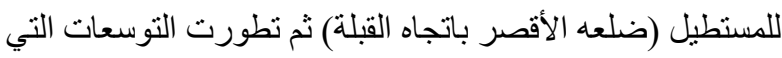

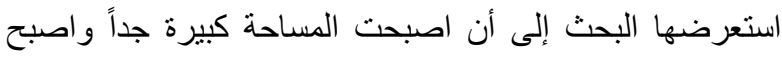

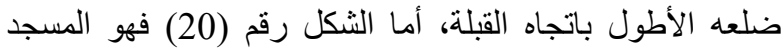




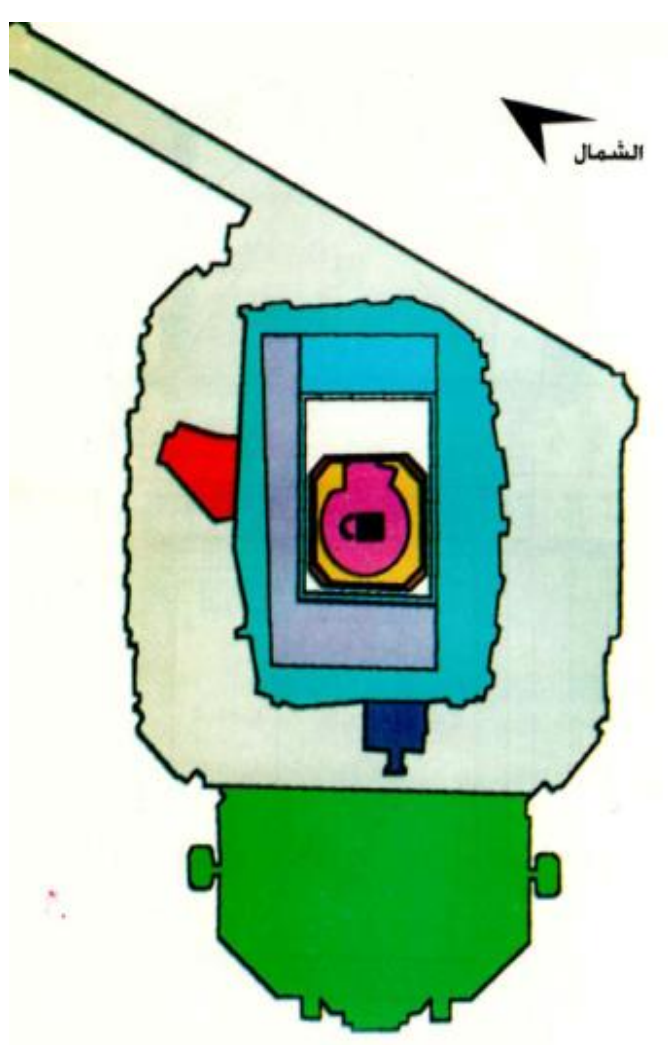

شكل رقم (20) - (20)

يوضح مخطط توسعة المسجد الحرام عبر التاريخ المصدر : خلوصي، 1998م الكسجر

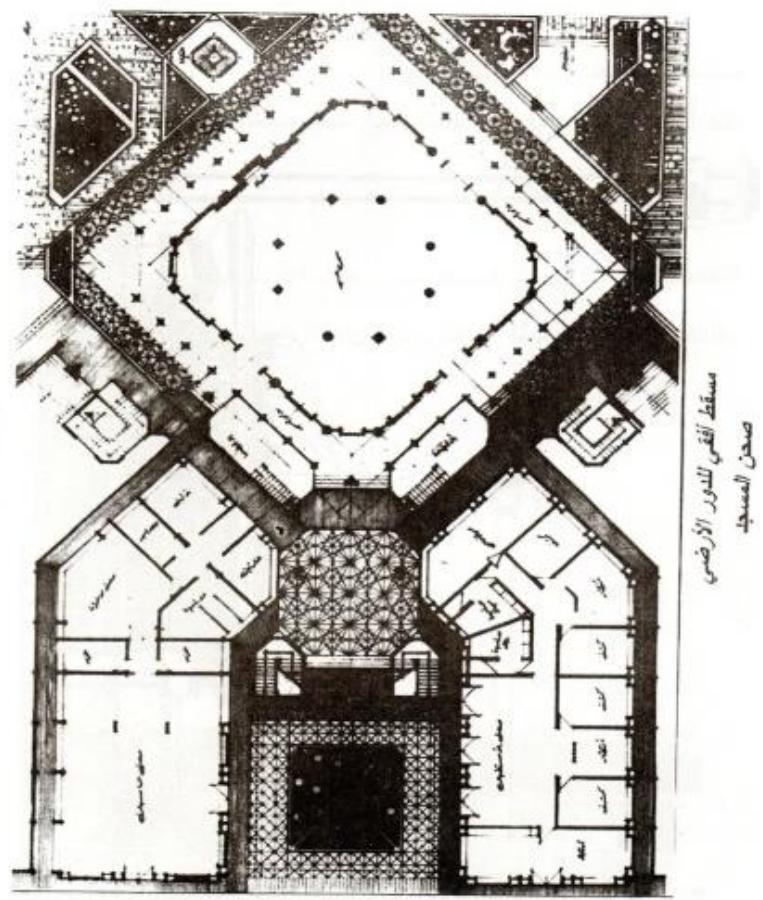

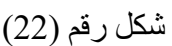

$$
\begin{aligned}
& \text { مسجد الرحمن - الثيخ غر اب - جمهورية مصر } \\
& \text { تصميم المهنس/ حسن رشوان } \\
& \text { المصدر خلوصي } 1998
\end{aligned}
$$

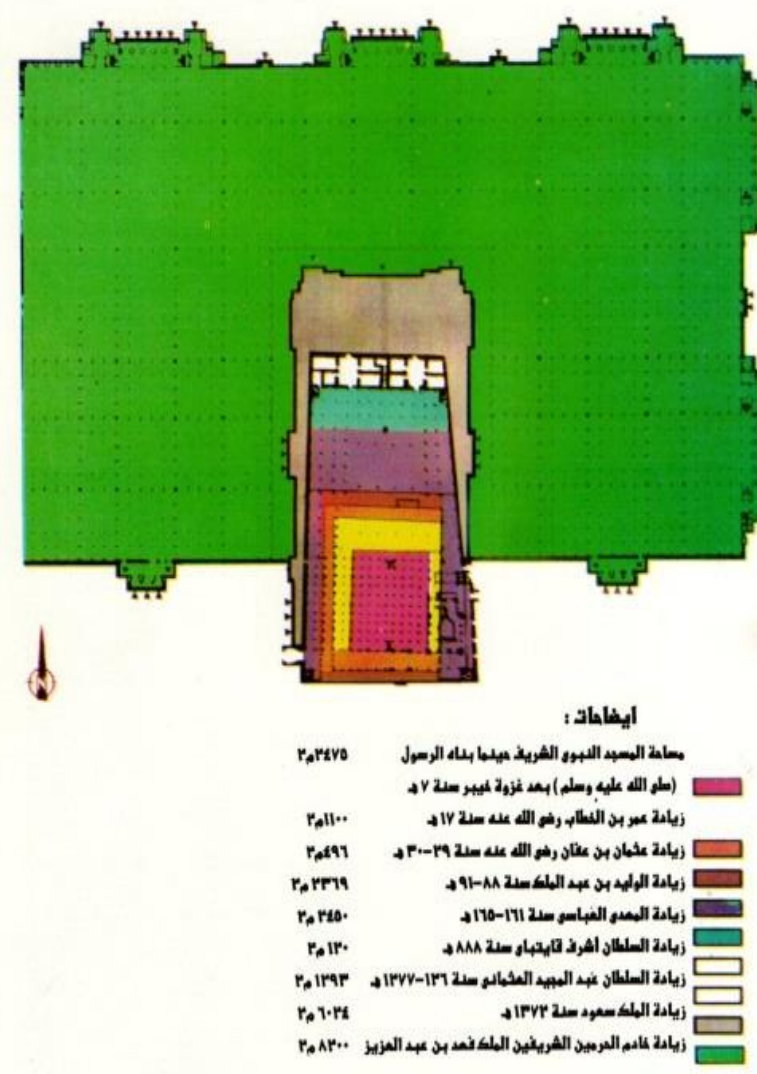

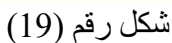

يوضح مخطط جميع التوسعات بالحرم النبوي الثريف المصدر: خلوصي، 1998م الترسعات بالترمي

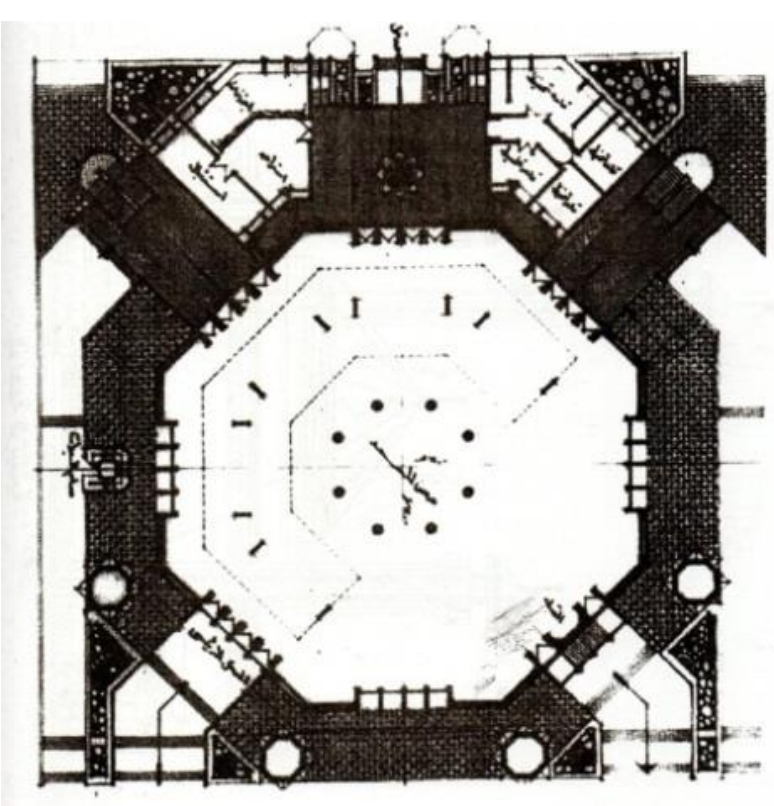

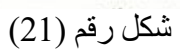

تصميم المهندم/ - مدينة نشوان 


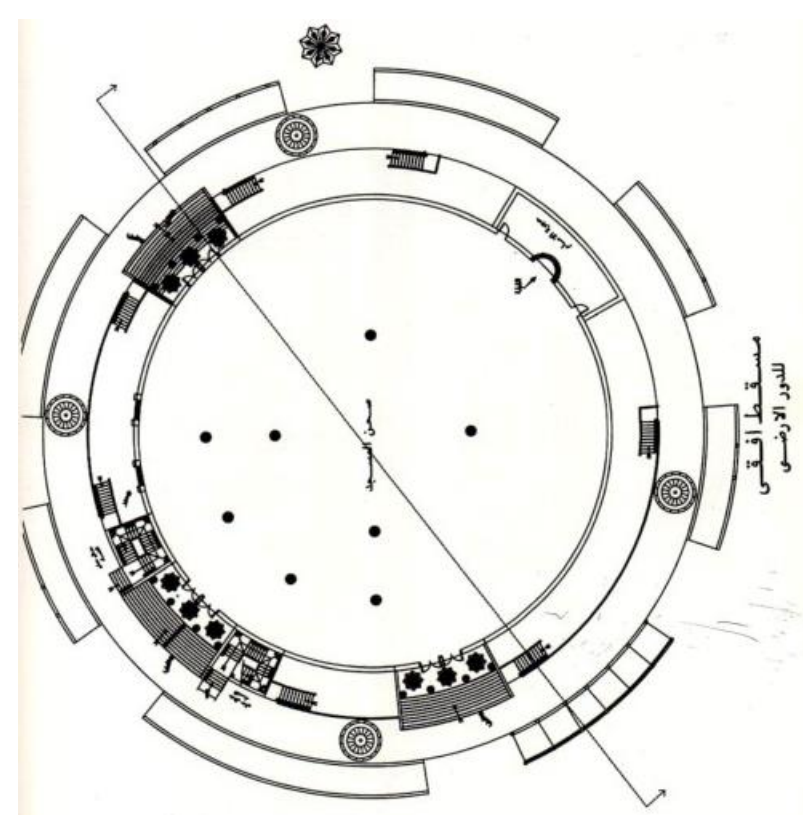

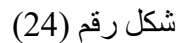

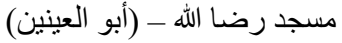

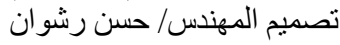

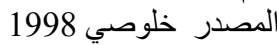

وسطبة (إلا التي تحمل طابق النساء) وبمسافات 25متر

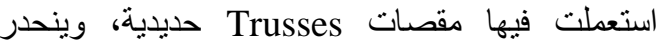

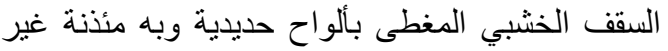

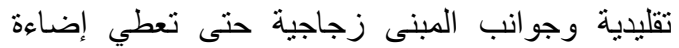

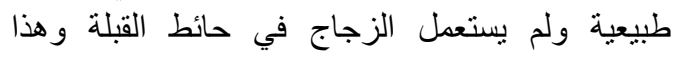

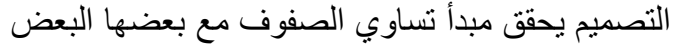

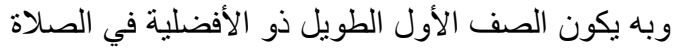

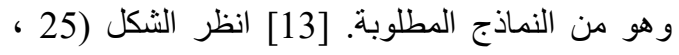

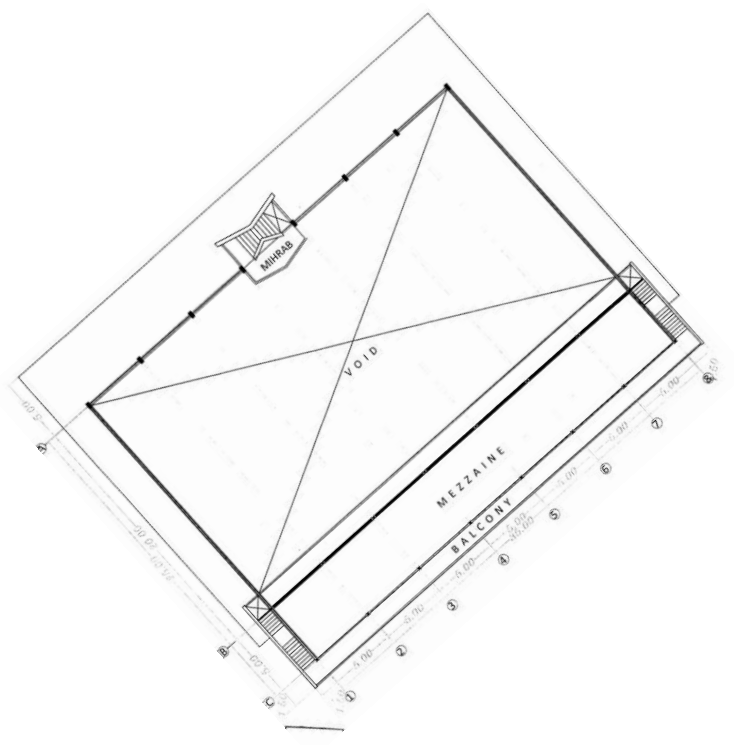

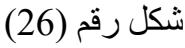

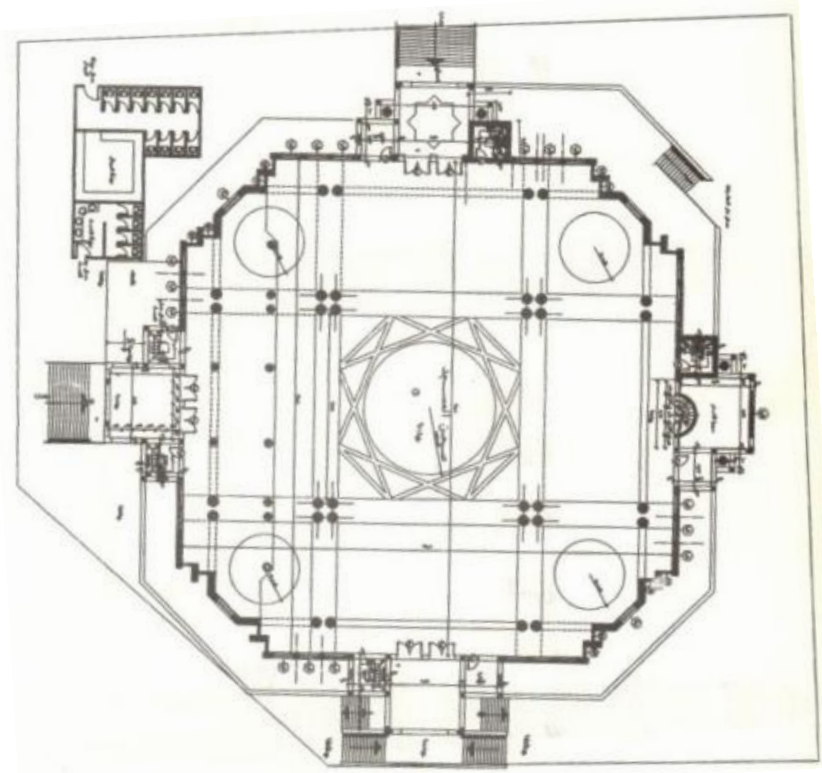

$$
\begin{aligned}
& \text { شكل رقم (23) } \\
& \text { مسجد الحصري - القاهرة } \\
& \text { تصميم الدكتور / هثام فتحي الفي } \\
& \text { الدصدر خلوصي } 1998
\end{aligned}
$$

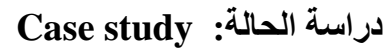

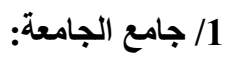

الموقع بالخرطوم شارع الجامعة على مساحة 1225

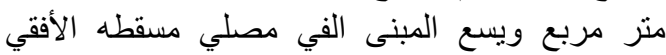

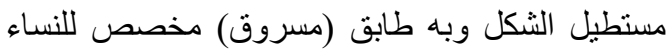
مستطيل الثكل أيضاً وقد صممه البانثمهندس محمد محمود حمدي بباحات خارجية واسعة بها مظلات على البه شكل Mushroom وفراغ داخلي كبير بدون أعمدة

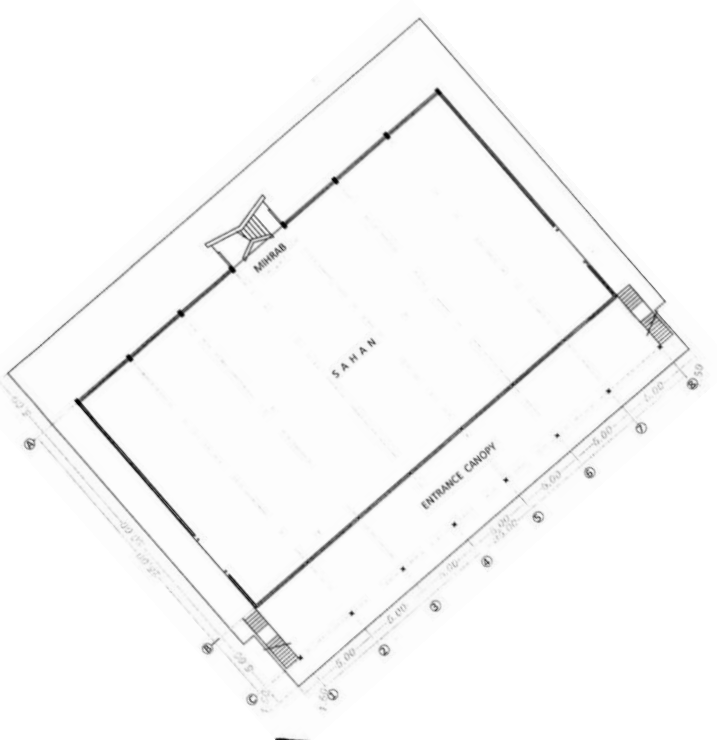

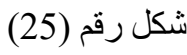

خارطة المسقط الأفقي - جامع الجامعة - الخرطوم

المصدر: شركة حمدى الاستثارية 
بالألوان، المسجد يعد تحفة معمارية متفردة، لكن شكل مسقطه الدائري تكون فيه صفوف الصلاة غير متساوية الأطوال و الصف الأول والذي به الأفضلية في الصلاة يكون أقصر الصفوف طو لاً. انظر الثكل رقم (27)

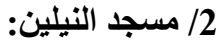

يقع بمدينة أم درمان - مسقطه الأفقي على شكل دائرة قطر ها

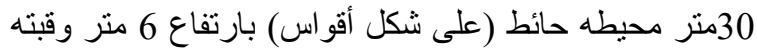

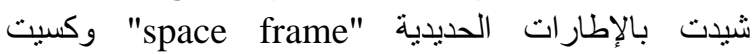
بالألمنيوم من الخارج ونقشت وزخرفت داخلياً، أخشابها

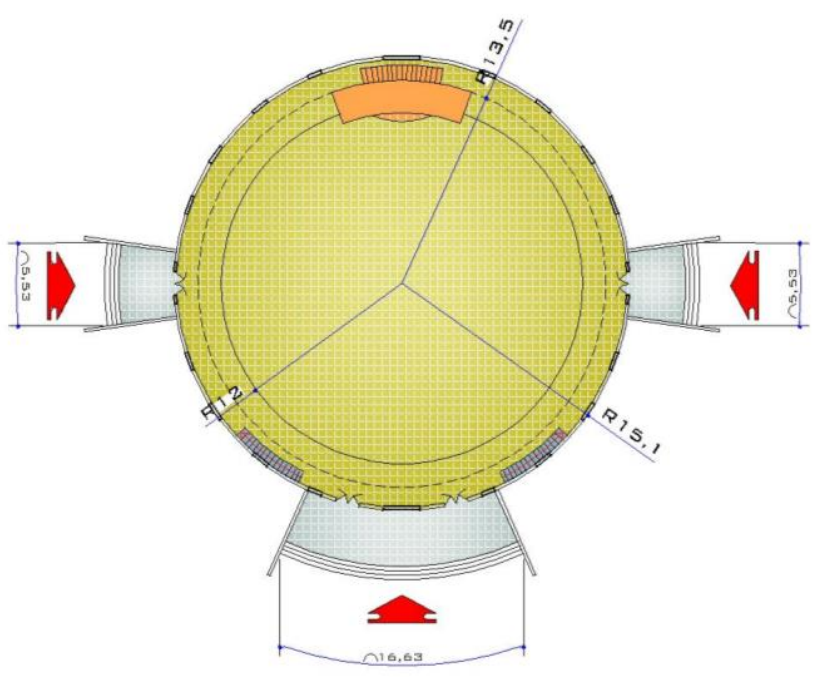

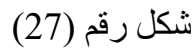

خارطة لمسجد النيلين_الطابق الارضي

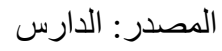

الصالة تستو عب كل الفعاليات السابق ذكر ها لذلك فانها يمكن أن

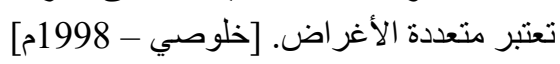
للمسجد معايير هامة عند التفكير في تصميمه يجب التأل التأكيد عليها،

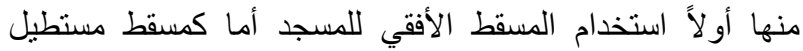

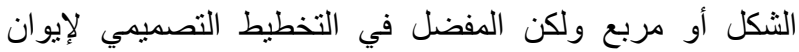
الصلاة المسقط الأفقي المستطيل، ويلاحظ أن الضلع الفيلع الأطول

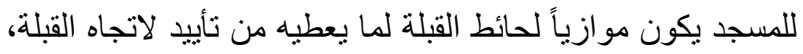
ويتم نوجيه ايو ان الصلاة في هذا الاتجاه. [زينهم - 2006م].

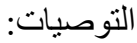

توصي الدراسة بالالتز ام و التقيد بتصميم وتتفيذ شكل المسقط الأفقي

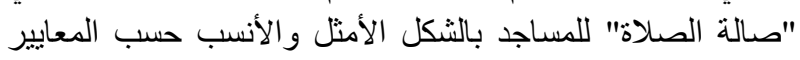

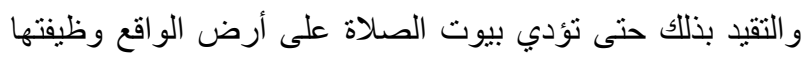

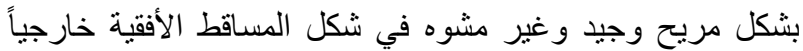

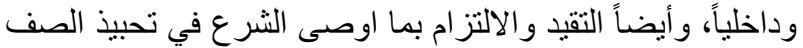

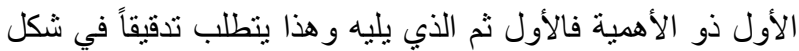

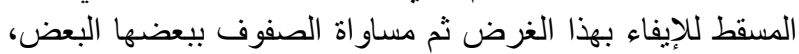
ويعنى بهذا الصف الأول ثم الذي يليه فالأوسط وحتى الأخير كلها لألها

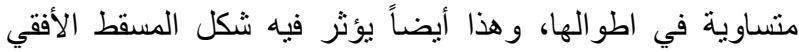

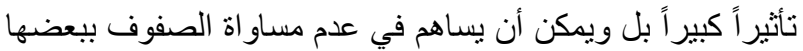

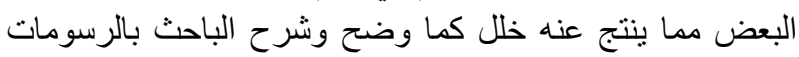
و الأشكال المرفقة وتحليلها.

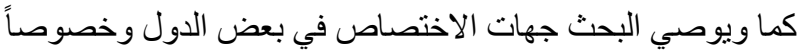
السودان للاهتمام بضبط قو انين البناء للمساجد وتفاصبلها.

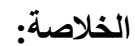

بعد استعر اض وتحليل هذه الأشكال تخلص الدراسة إلى أنه من الأن الهاء

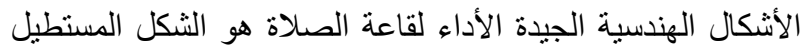

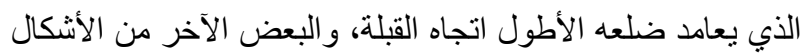

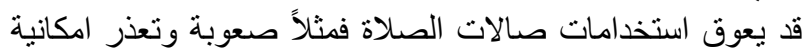

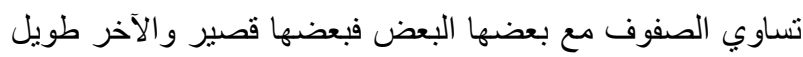

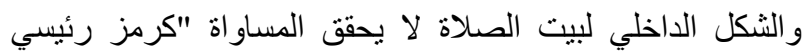

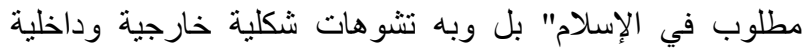

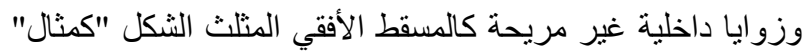

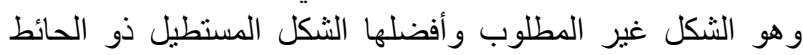

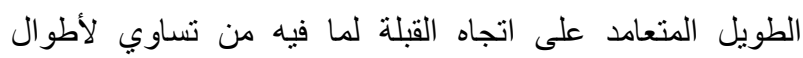

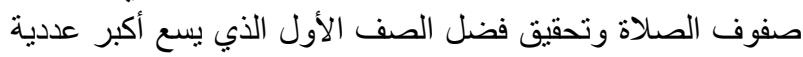

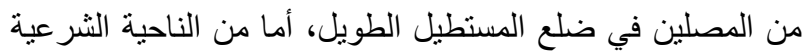

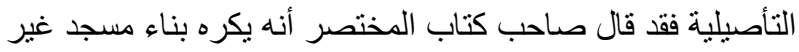

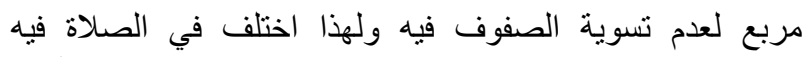

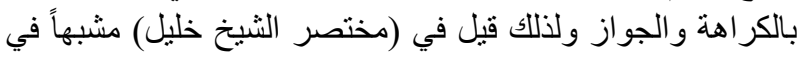

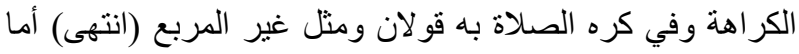

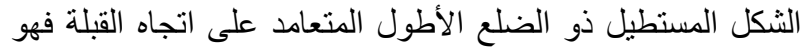

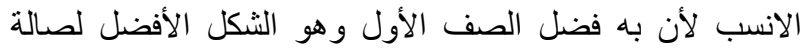

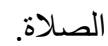
و المصلى هو القسم الرئيسي في المسجد ففي المسجد تقام الصلاة

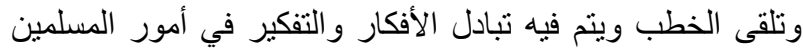

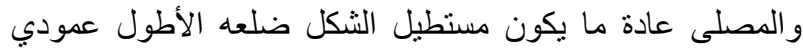
على اتجاه القبلة، وضلع القبلة هذا يضم المحراب و المنبر وهذا 
3. مهنس مستثار، خلوص، محمد ماجد عباس، المسجد عمارة وطر از وتاريخ،

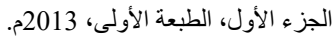

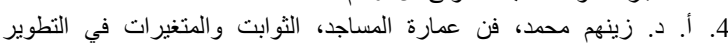

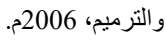

5. حسن، نوبي محمد، خصائص التفكير في تصميم الحيز الداخلي للمساجد، أبحاث

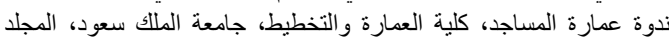

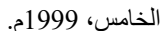

6. أحمد فكري: مسجد الزيتونة الجامع في تونس، المجلة الناريخية المصرية،

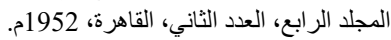

7.

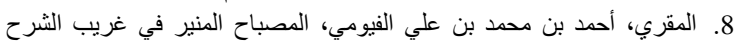
دالكبير، مكتبة لبنان، طبعة 2009م، الثبكة العنكبوتية. 9. د. رزق عاصم محمد، معجم المصطلحات العمارة والفنون الإسلامية، مكتبة لإنة

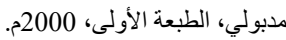
10. خليل بن اسحاق الجندي المالكي، مختصر خليل، دار الفكر، نشر 2010م،

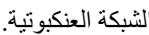

11. النووي محي الدين بن يحي، رياض الصالحين.

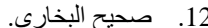
13. شركة حمدي الاستثارية، مهنس محمد محمود حمدي. 14. أبن حبان، الحافظ محمد، صحيح ابن حبان، دار المعارف، دئدئ 1952. 